\title{
A Survey on Recent Advances in Organic Visible Light Communications
}

\author{
Zahra Nazari Chaleshtori ${ }^{1}$, Petr Chvojka ${ }^{1}$, Stanislav Zvanovec ${ }^{1}$, Zabih Ghassemlooy ${ }^{2}$ and Paul Anthony Haigh ${ }^{3}$ \\ ${ }^{1}$ Dept. of Electromagnetic Field, Czech Technical University in Prague, Prague, Czech Republic, 16627 \\ ${ }^{2}$ Optical Communications Research Group, Faculty of Engineering and Environment, Northumbria University, Newcastle-upon-Tyne, NE1 8ST, \\ $U K$ \\ ${ }^{3}$ Communications and Information Systems, University College London, London, WC1E 6BT, UK \\ \{nazarzah; chvojpe8; xzvanove\}@fel.cvut.cz,z.ghassemlooy@northumbria.ac.uk,p.haigh@ucl.ac.uk
}

\begin{abstract}
Visible light communication (VLC) employs light emitting diodes (LEDs) to provide illumination and data communications simultaneously. Organic LEDs (OLEDs) employing small molecules and long-chain polymers PLEDs, have been gaining attention within the VLC research community due to their inherent advantages such as flexible substrates and low-cost manufacturing. However, the carrier mobility of organic semiconductors is much slower than the devices composed of metal alloys, such as gallium nitride, thus leading to a restriction in the OLED modulation bandwidth. The manufacturing processes, materials and the photoactive size of the devices can affect the raw bandwidth of OLEDs. To increase the transmission speeds, novel approaches have been proposed including equalization techniques, signalling schemes and the optimum driver circuits. The paper provides a survey on the evolution of OLED-based VLC systems, and the respective challenges and recent progresses.
\end{abstract}

Keywords-Visible light communication; Organic LEDs; Optical channel; Equalizers.

\section{INTRODUCTION}

Visible light communications (VLC) is a new wireless technology for transmitting data by means of intensity modulation of light emitting diodes (LEDs). VLC offers advantages over the radio frequency (RF) technologies such as inherent security, immunity to RF-based electromagnetic interference, license-free spectrum and high scalability [1, 2]. LEDs are based on both inorganic and organic materials, with the latter being explored for a range of applications [1]. LEDs produce white light with either a package that contains individual red, green, and blue (RGB) LED chips or using a blue LED that has a cerium-doped yttrium aluminium garnet (Ce:YAG) yellowish phosphor encompassing the photoactive area, known as white phosphor LEDs (WPLEDs) [3]. The wide spread use of LED lights at home, offices, streets, and public places, acts as a good motivator for utilisation of VLC for indoor and outdoor applications [1]. For example, in airplanes, hospitals, intelligent transportation systems [4], indoor localization $[5,6]$, machine-to-machine communications or the internet of things (IoT) [7], and underwater communications [8].

Organic LED (OLED)-based VLC (OVLC) is an emerging subset of VLC that uses organic photonic devices as the transmitter (Tx), the receiver ( $\mathrm{Rx})$ or both. The organic materials can be long-chain polymers (PLEDs) or small organic molecules (SMOLEDs) in a crystalline phase $[9,10]$. SMOLEDs and PLEDs have been the focus of increasing research in recent years, due to a number of advantages including: $(i)$ improved image quality - better contrast, higher brightness, fuller viewing angle, a wider colour range, transparent, and much faster refresh rates; (ii) lower power consumption; (iii) simpler design, which enables ultra-thin, flexible, foldable and transparent displays; (iv) improved durability - can operate in a broader temperature range; and ( $v$ ) large photoactive areas $[1,2]$. As a result, OLEDs have been gaining interest in the fields of solid state lighting [11], display technology [12] and optical wireless broadcasting networks [13-15]. Obviously, thanks to the merits of OLED, the OVLC systems have tremendous advantages over VLC systems including transparent and large photoactive display, usage on bent surface, operation in a broader temperature range, lower power consumption. Recently, organic photodiodes (OPDs) are seen as a next generation device for optical communications and image sensor-based applications due to their high sensitivity and superior responsivities [16]. With organic devices having a relative low bandwidth $B_{\text {od }}(<1 \mathrm{MHz})$, the vast majority of research works on OVLC has focused on increasing the data rate $R_{b}$, which is the topic of review in this paper.

The rest of the paper is organized as follows. In Section II, organic components are introduced. In Section III optical channel modelling and in Section IV review on recent advances are discussed. Finally, Section V outlines the conclusion.

\section{ORGANIC COMPONENTS}

\section{A. Charactrization of Organic LEDs}

A semiconductor is considered organic if the materials are mainly carbon or nitrogen [17]. Organic materials possess similar characteristics to metallic semiconductors and polymers concurrently [18]. These features are attractive for the emerging new type of electronics industry - organic electronics. The most popular devices are OLEDs and organic solar cells known as organic photovoltaics (OPV). Organic devices are based on thin film technology; the general structure for a photonic device consists of two or more organic semiconductor materials sandwiched by oppositely polarized electrodes, see Fig. 1.

The total stack thickness of an OLED is between 100-500 $\mathrm{nm}$ depending on the manufacturing process, which is ideal for future displays [19]. Furthermore, having large photoactive areas and a reduction in processing costs provide an important benefit, because of increasing popularity of thin film devices in 


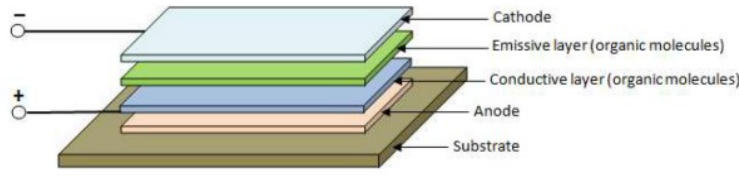

Fig. 1. OLED structure

modern technologies such as mobile phones, TVs and lighting fixtures. OLEDs exhibit capacitor-like behaviour with a lowpass filter transfer function with a cut-off frequency given by [1, 20]:

$$
f_{s}=\frac{1}{2 \pi R C}
$$

where $R(\Omega)$ is the effective resistance of the OLED and $C$ is the plate capacitance expressed as [1]:

$$
C=\frac{A \varepsilon_{0} \varepsilon_{r}}{d}
$$

where $A\left(\mathrm{~m}^{2}\right)$ is the OLED thickness and $d(\mathrm{~m})$ the OLED photoactive area, $\varepsilon_{0}(\mathrm{~F} / \mathrm{m})$ and $\varepsilon_{r}$ (unit-less ratio) are the permittivity of free space and relative dielectric constant of the organic layer, respectively. Several important materials have been used for the electron injection layer of OLEDs such as $\mathrm{LiF}$, $\mathrm{NaCl}, \mathrm{NaF}$ and their dielectric constants are 9.036, 5.895 and 5.072 , respectively at the temperature of $300 \mathrm{~K}$ [21].

From (2) $C$ is proportional to the photoactive dimensions and hence, a large photoactive area, which leads to a low modulation bandwidth (BW) (to around a few hundreds $\mathrm{kHz}$ [22]) not desirable in OVLC. Additionally, bandlimited systems experience degradation in the bit error rate (BER) performance due to inter-symbol interference (ISI). The baseline wander (BLW) phenomenon is another challenge in OVLC systems, where the signal randomly deviates from the DC level due to high pass filtering or capacitive coupling. A number of methods have been proposed to overcome the small $B_{\text {od }}$ and ISI including (i) high-level modulation schemes with no equalization or raised cosine filtering [14, 23], which are the most popular methods of mitigating ISI; and (ii) equalization such as the artificial neural network (ANN) based equalizer to maximize $R_{b}$ by undoing the detrimental effect of the ISI [13, 24]. In [25] it was experimentally demonstrated that the radiation pattern of a bent OLED panel is not Lambertian, and an improved analytic mixed Gaussian model was proposed to describe the rotational radiation asymmetry, where parameters values were found by using an expectation-maximization algorithm for curve fitting with the measured data. Compared with Lambertian source, OLED sources are more flexible in radiation pattern control showing advantages in terms of lower root mean square delay spread and path loss. However, its impact on the VLC channel characteristics has not been investigated.

\section{B. Organic photodiodes}

An OPD is a semiconductor device that converts light into an electrical current. OPDs may contain optical filters, built-in lenses, amongst other optics [26]. Due to the aforementioned advantages of OPDs, including flexibility and high responsivity, they have gained attention in recent years [16]. Since OPDs offer higher responsivity compared to silicon ( $\mathrm{Si}$ ) PDs in the visible spectrum, they are ideal in VLC systems [27]. In many organic optoelectronic devices, including OLEDs, OPVs and OPDs, a hole injection layer (interlayer) is usually inserted between the transparent anode and the active layer as electron blocker. Several strategies are reported in the literature to improve hole extraction from the photoactive layer. The most popular is incorporation of several different types of hole extraction layers in the device stack such as conductive polymeric materials, selfassembled molecules and metal oxides, surface treatment of the positive electrodes and the conductive polymeric layers [28]. The interlayer at the negative electrode can improve the OPD performance, transferring electrons more efficiently and blocking the movement of holes from the active layer to the cathode. Furthermore, although the local vacuum reference energy level is assumed to be constant at each interface in the organic device, it seems that the use of an interlayer can result in a device with interface dipoles at the organic/metal and organic/organic interfaces, thus resulting in a shift of the vacuum level [28]. This indicates that, the interlayer plays an important role in the charge injection/extraction control, since it can set the work function of the electrode.

\section{PRINCIPLE OF VLC}

In order to model the optical channel in VLC, a number of possible link configurations can be considered: $(i)$ directed lineof-sight (LOS) where the LED is aligned directly with the Rx with no offset in order to minimize BER; and (ii) non-LOS (NLOS), i.e., the signal is received via reflections from walls and ceiling [29]. Fig. 2 illustrates a block diagram of a typical VLC link. The desired modulation format is applied to the data generated by The pseudorandom binary sequence (PRBS) data, which can be created either by MATLAB or by a field programmable gate array (FPGA) using shift registers, is modulated prior to intensity modulation of the light source via the driving circuit. A DC bias includes to meet the optical power constraint. Following transmission over the wireless channel the incoming optical signal is captured by a PD, and the regenerated electrical signal is given as:

$$
y(t)=\Re G x(t) \otimes h(t)+n(t)
$$

where $h(t)$ is the channel impulse response, $\mathfrak{R}$ and $G$ are the detector responsivity and gain, respectively, the $\otimes$ symbol denotes convolution, and the noise $n(t)$ is mainly due to the ambient light and in the form of shot noise [1]. The main sources of ambient noise are sunlight and artificial light. During daytime, sunlight through windows is typically stronger than the other noise sources. The dominant noise source $n(t)$ in VLC links is the background light induced shot noise. With a low level of ambient light, the dominant noise source is the thermal noise. The additive white Gaussian noise (AWGN) model can be adopted with single sided power spectral density $N_{0}$ [30]. The signal is demodulated and processed to determine the BER performance, the error vector magnitude (EVM) and/or the signal-to-noise (SNR) ratio.

\section{A. Illuminance of LEDs}

Assuming that the source has a Lambertian radiation pattern, which is given in terms of the spatial angle $\omega$ and the luminous flux $\Phi$ as [1]: 


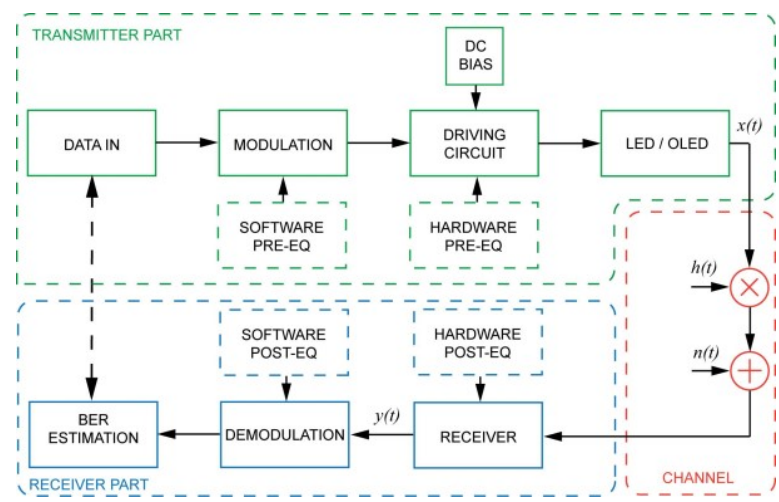

Fig. 2. A block diagram of a typical OVLC link

$$
I=d \Phi / d \omega
$$

The intensity of light is a continuous signal $x(t)$ with an amplitude which is dependent on a modulation scheme. The average power $P_{\text {avg }}$ is given by [31]:

$$
P_{a v g}=\frac{1}{2 T} \int_{-T}^{T} x(t) d t
$$

The luminous intensity defined in terms of the angle of irradiance $\phi$ is given as [32]:

$$
\mathrm{I}(\phi)=\frac{m+1}{2 \pi} \mathrm{I}(0) \cos ^{m}(\phi), \phi \in\left[-\frac{\pi}{2}, \frac{\pi}{2}\right] .
$$

where $I(0)$ is the centre luminous intensity of the LED, $\phi$ is the angle of irradiance and $m$ is Lambertian order, given in terms of the Tx semi-angle $\phi_{1 / 2}$ as $[31,32]$ :

$$
m=-\frac{\ln (2)}{\ln \left[\cos \left(\phi_{1 / 2}\right)\right]} \text {. }
$$

Note that, only for $m=1\left(\phi_{1 / 2}=60^{\circ}\right)$ the source is Lambertian, whereas for $m>1$ the source becomes increasingly directed. To maximize luminous intensity, $\phi=0^{\circ}$ and hence, a directed LOS link will provide the best signal quality [1].

\section{B. Channel Model}

To simplify the channel model, here we consider only the LOS configuration. The channel can be described either in terms of frequency response as $[1,31]$ :

$$
H(f)=\int_{-\infty}^{\infty} h(t) \mathrm{e}^{-2 \pi f t} d t .
$$

The received optical power $P_{R}$ is given by [31]:

$$
P_{R}=H(0) P_{E}
$$

where $P_{E}$ is the emitted optical power, and the channel DC gain $H(0)=\int_{-\infty}^{\infty} h(t) d t$. For a generalized Lambertian pattern, the impulse response of the LOS channel is given by [1]:

$$
H_{L O S}(0)=\frac{A_{r}}{d^{2}} \mathrm{I}(\phi) \cos (\varphi) \mathrm{T}_{s}(\varphi) g(\varphi), 0 \leq \varphi \leq F O V
$$

where $A_{r}$ is the PD surface area, $d$ is the distance from LED to $\mathrm{PD}, \varphi$ is the angle of incidence on the $\mathrm{PD}, T_{s}(\varphi)$ is the optical filter gain, and the optical concentrator gain $g(\varphi)$ is given as [1]:

$$
g(\varphi)=\frac{n^{2}}{\sin ^{2} \varphi_{c}}, 0 \leq \varphi \leq F O V
$$

where $n$ is the concentrator refractive index, and $\varphi_{c}$ is the field of view (FOV) of Rx.

\section{Modulation Schemes}

There are two typical groups of modulation schemes for optical communications: ( $i$ ) baseband modulations; and (ii) multi-carrier modulations. The most popular baseband schemes are on-off keying (OOK), pulse position modulation (PPM) or pulse amplitude modulation (PAM) [1]. The multi-carrier modulations include orthogonal frequency division multiplexing (OFDM) and carrier-less amplitude and phase (CAP) modulation [27]. Since OVLC systems are highly bandlimited, a modulation scheme is desirable that can transmit more symbols-per-bit within a small $B_{\text {od. }}$ In addition, since LEDs have dual functionality of illumination and data communications, then modulation schemes adopted should also offer dimming control. A number of modulation schemes have been developed with this features such as multiple PPM (MPPM) [33, 34]. Furthermore, analysing the communications link performance in terms of power requirement and spectral efficiency $\eta_{s}$ is essential. The most power efficient scheme is the high order $L$ PPM, but requires the largest bandwidth [19]. In [35] it is shown that variable OOK (VOOK) requires the same power as variable PPM (VPPM) while its $\eta_{s}$ is better than VPPM. In addition, under $50 \%$ brightness, return-to-zero (RZ) OOK should require $3 \mathrm{~dB}$ less power than VOOK with $\eta_{s}$ of $1[\mathrm{~b} / \mathrm{s} / \mathrm{Hz}]$; however, RZOOK provides at most $50 \%$ brightness. The technique of optical-fast OFDM (O-FOFDM) reported in [36] aims to improve $\eta_{s}$ by using half the bandwidth of OFDM, with the caveat that it is limited to one dimensional modulation formats. To achieve optical bandwidth saving, a dense OFDM (DOFDM) is employed by narrowing the spacing between two optical subchannels in [37], while [38] proposed a higher number of subchannels using Nyquist wavelength division multiplexing (NWDM) techniques. In [39] 50\% electrical bandwidth saving is achieved using a mix of electrical and optical single sideband signal manipulation techniques. In addition, an optical spectrally efficient frequency division multiplexing (O-SEFDM) system was proposed in [40] to provide higher $\eta_{s}$ relative to O-OFDM, where non-orthogonal and overlapping sub-carriers are employed so that the performance is the same as O-OFDM for bandwidth saving up to $25 \%$. The O-SEFDM technique can also increase $\eta_{s}$ in both electrical and optical domains. The CAP scheme was shown to outperform OFDM in terms of $R_{b}$ using the same physical link in VLC [41]. CAP systems have several advantages over OFDM, a single carrier modulation is utilized rather than Fourier transform $[42,43]$. In $m$-CAP the signal bandwidth is split into several subcarriers, where the attenuation caused by an LED frequency response is decreased. Therefore VLC links by allocating different bandwidths for individual subcarriers, with high $\eta_{s}$ (i.e., up to $36 \%$ improvement in $R_{b}$ for 6-CAP) can be supported [44]. The first VLC experiment utilizing $m$-CAP was reported in [43] with $R_{b}$ of $31.5 \mathrm{Mb} / \mathrm{s}$ using 
10-CAP; thus resulting in $\eta_{s}$ of $4.85 \mathrm{bps} / \mathrm{Hz}$, which offers huge potential for a future research.

Since the nonlinearity of the source degrades the system OVLC performance, therefore transmitting an OFDM signal results in intermodulation products between the subcarriers and an ICI [1]. In addition, giving that OFDM signals have a relatively high dynamic range, which is described often in the terms of the peak-to-average power ratio (PAPR), subcarriers can add constructively or destructively, thus leading to a large variation in the transmit signal power level [1]. High PAPR means large saturation power for power amplifiers, which results in reduced power efficiency. To combat this, PAPR reduction techniques of signal scrambling and signal distortion (i.e., companding, signal clipping, peak windowing, envelope scaling, and peak reduction carrier) can be adopted [1]. E.g., for OFDM-VLC in [45] an exponential nonlinear companding transformation techniques and in [46, 47] an advanced A-law companding algorithm were proposed. Alternative, in [48] a hybrid modulation scheme was proposed where the OFDM signal is converted to a pulse width modulation format prior to intensity modulation of the light source in order to mitigate the requirement for higher PAPR.

\section{System Performance}

For intensity modulation/direct detection (IM/DD) optical transmission systems, the electrical SNR is defined as [31]:

$$
S N R=\frac{\left(\Re P_{R}\right)^{2}}{R_{b} N_{0}}=\frac{\Re^{2} H^{2}(0) P_{E}^{2}}{R_{b} N_{0}}
$$

To evaluate quality of the link, BER is measured, either by $Q$ factor or by exact comparisons between transmitted and received data [32]. The $Q$-factor is given by [49]:

$$
Q=\frac{v_{H}-v_{L}}{\sigma_{H}+\sigma_{L}}
$$

where $v_{H}$ and $v_{L}$ are the mean received voltages, $\sigma_{H}$ and $\sigma_{L}$ are the standard deviations of the 1-level and 0-level signals before being sliced, respectively. The BER for OOK modulation is defined as [49]:

$$
B E R=\frac{1}{2} \operatorname{erfc}\left(\frac{Q}{\sqrt{2}}\right)
$$

\section{REVIEW ON RECENT ADVANCES OF OVLC}

As stated earlier, the major challenge in using organic-based optoelectronic devices in VLC systems is their very low $B_{\text {od, }}$, which limits the maximum data that can be transmitted. Therefore, signalling schemes, multi-level modulations, and equalization techniques have been used to overcome the bandwidth limitation. It necessitates the optimal design of the electronics, e.g., the driver circuit for the OLED, and the characterization of the organic components.

Fig. 3 shows the achieved $R_{b}$ in OVLC for different optical components, modulation schemes and equalizers. In [32] an experimental OOK-OVLC link using a SMOLED ( $B_{\text {od }}$ of 93 $\mathrm{kHz}$ ) and a silicon PIN-PD as the Tx and the Rx, respectively with an ANN equalizer at $R_{b}$ of $550 \mathrm{~kb} / \mathrm{s}$ with no errors was reported. A $1.4 \mathrm{Mb} / \mathrm{s}$ OVLC system using an OLED ( $B_{\text {od }}$ of 93 $\mathrm{kHz}$ ) with discrete multitone (DMT) modulation was experimentally demonstrated in [50]. In [24] an OVLC using an OLED with $B_{\text {od }}$ of $150 \mathrm{KHz}$ and an $\mathrm{RC}$ equalizer $R_{b}$ was increased to $2.15 \mathrm{Mb} / \mathrm{s}$. In [3] it was demonstrated that by isolating the $\mathrm{AC}$ data source using a high impedance NAND gate (i.e., no low frequency restrictions and therefore no BLW effect) $R_{b}$ can be increased. In addition, the modulation depth was increased from $<10 \%$ using a bias-tee up to $\sim 100 \%$, thus significantly improving the SNR. In [3] it was shown that $R_{b}$ can be increased significantly using the multilayer perceptron (MLP)-ANN to 2.7, 2.2, and $1.25 \mathrm{Mb} / \mathrm{s}$ for 4-PPM, OOK, and 2-PPM, respectively compared to the non-equalized system.

Alternatively, multi-carrier modulation techniques such as OFDM have been adopted to increase the $R_{b}$. In [51] it was shown that, $3 \mathrm{Mb} / \mathrm{s}$ can be achieved using OFDM- quadrature amplitude modulation (QAM) based OLED with a $93 \mathrm{kHz}$ bandwidth. In several recent works, new experimental results on PLED-based VLC have been reported. For instance, in $[52,13]$ a Si- PD based Rx was adopted and achieved $10 \mathrm{Mb} / \mathrm{s}$ and 20 $\mathrm{Mb} / \mathrm{s}$ for OOK OVLC with a least mean squares (LMS) equalizer (PLED of $270 \mathrm{kHz}$ ) and an ANN equalizer (PLED of $350 \mathrm{kHz})$, respectively. The former system with OFDM increased $R_{b}$ to $10 \mathrm{Mb} / \mathrm{s}$ with no equalization $[14,23]$. Furthermore, in [53] based on wavelength-division multiplexing (WDM) using three individual RGB pixels of a PLED and an ANN equalizer $R_{b}$ per PLED chips of $27.9 \mathrm{Mb} / \mathrm{s}, 18.6 \mathrm{Mb} / \mathrm{s}$ and

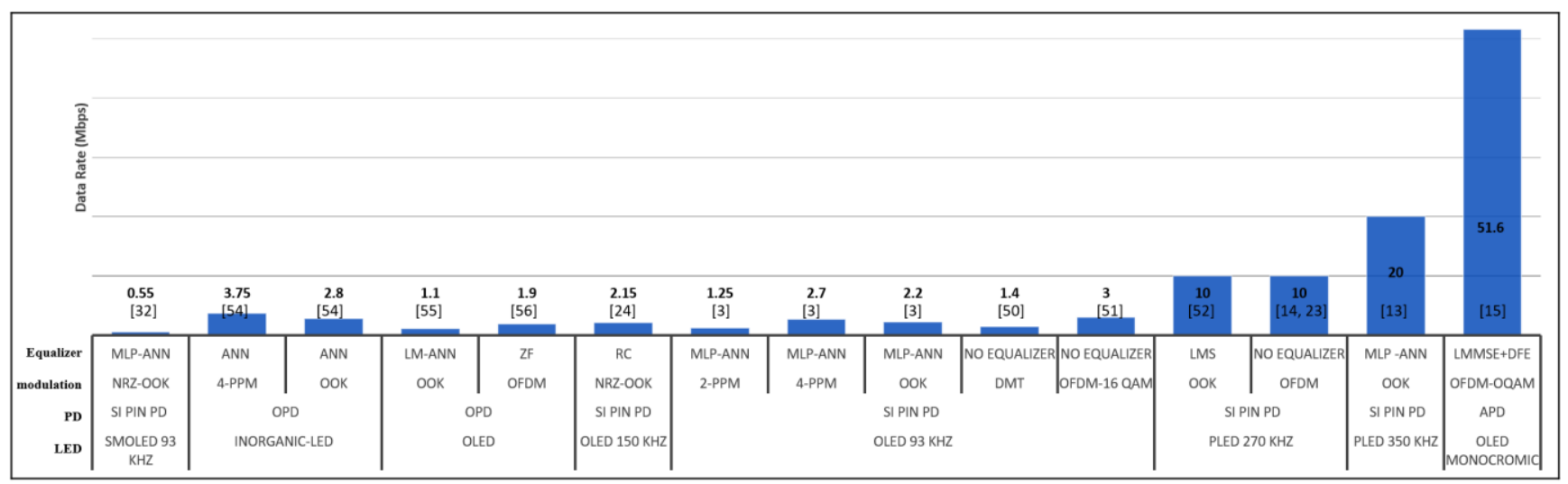

Fig 3. The evaluation of the achieved data rates in OVLC 
$8.4 \mathrm{Mb} / \mathrm{s}$ for the red, blue and green components, respectively (i.e., an aggregate link capacity of $\sim 55 \mathrm{Mb} / \mathrm{s}$ ) were reported.

OPDs are adopted in VLC systems, since they can offer superior responsivity in comparison to Si-PDs in the visible spectrum. E.g., in [54] a VLC system employing an inorganicLED, OPD of $160 \mathrm{kHz}$, and an ANN equalization achieved $R_{b}$ of 2.8 and $3.75 \mathrm{Mb} / \mathrm{s}$ for NRZ OOK and 4-PPM, respectively. In [55], an all-organic-based VLC employing an ANN equalizer with Levenberg-Marquardt (LM) demonstrated a $R_{b}$ of $1 \mathrm{Mb} / \mathrm{s}$ over a short transmission span. Whereas, in [56] an OFDMbased all-organic VLC link with a bit/power loading algorithm to combat the system frequency selectivity reported $R_{b}$ of 1.9 $\mathrm{Mb} / \mathrm{s}$. In [22] it was demonstrated that setting a specific carrier frequency $f_{c}$ can affect the system performance. The achieved $R_{b}$ of 220,640 and $260 \mathrm{~kb} / \mathrm{s}$ for $f_{c}$ of 100,200 and $300 \mathrm{kHz}$, respectively was reported. Also investigated was the angular dependence of the system performance showing $R_{b}$ of $160 \mathrm{~kb} / \mathrm{s}$ with a tilt angle up to $48^{\circ}$. Thus, opening up the possibility of adopting advanced techniques, e.g., the angle diversity.

Recently, $51.6 \mathrm{Mb} / \mathrm{s}$ OLED based VLC employing a monochromic OLED fed by an OFDM signal with offset QAM (OQAM) has been reported [15]. In this work, the bit and power loading technique and a joint linear minimum mean-square-error (LMMSE) and a decision feedback equalizer (DFE) were used at the Rx to combat ISI, and a cyclic prefix (CP) to improve $\eta_{s}$. The work in [57] investigated the OLED nonlinearity using Volterra series and a Volterra-based nonlinear equalizer in order to mitigate both ISI and nonlinearity more effectively than other equalizers over a link span of $3 \mathrm{~m}$. Recently, organic solar cells have been used as high-speed detectors for VLC as well as supplying the power needed for the Rx circuit [58-60].

\section{CONCLUSION AND Future OUTLOOK}

In this paper the current status of OVLC was outlined considering different organic devices, channel model and modulation formats. We surveyed the recent OVLC systems reported in the literature showing a significant increase in the achievable $R_{b}$ from a few $\mathrm{kb} / \mathrm{s}$ to $>50 \mathrm{Mb} / \mathrm{s}$ using a single pixel based devices. Due to ever increasing momentum in the development of organic technology and their attractiveness in VLC used in certain applications is palpable. Based on the literature survey, we showed that OVLC will find application in two separate domains; (i) large single-drive display panels which can be produced easily, requiring novel drive circuits to compensate for the large capacitance; and (ii) pixels used in mobile devices - since OLED matrices can be produced cheaply on large scales. There is a significant potential to facilitate device-to-device communications via the display of mobile devices. In this respect, the advantages of OLED-based devices can go some way in addressing the challenges ahead. Both of these challenges will be explored in future work.

\section{ACKNOWLEDGMENT}

The work is supported by the European Union's Horizon 2020 research and innovation programme under the Marie Slodowska-Curie grant agreement no 764461 (VISION).

\section{REFERENCE}

[1] Ghassemlooy, Z., et al., "Visible Light Communications: Theory and Applications." 2017: CRC Press.

[2] Haigh, P.A., et al. "Organic visible light communications: Recent progress." in Transparent Optical Networks (ICTON), 2014 16th International Conference on. 2014. IEEE.

[3] Haigh, P.A., et al., "Visible light communications using organic light emitting diodes." in IEEE Communications Magazine, 2013. 51(8): p. 148-154.

[4] Luo, P., et al., "Undersampled-Based Modulation Schemes for Optical Camera Communications." in IEEE Communications Magazine, 2018. 56: p. 204-212.

[5] Lin, B., et al., "An indoor visible light positioning system based on optical camera communications." in IEEE Photonics Technology Letters, 2017. 29(7): p. 579-582.

[6] Armstrong, J., Y. Sekercioglu, and A. Neild, "Visible light positioning: a roadmap for international standardization." in IEEE Communications Magazine, 2013. 51(12): p. 68-73.

[7] Schmid, S., et al. "EnLighting: An indoor visible light communication system based on networked light bulbs." in Sensing, Communication, and Networking (SECON), 2016 13th Annual IEEE International Conference on. 2016. IEEE.

[8] Khalighi, M.-A., et al., "Underwater visible light communications channel modeling and system design, in Visible Light Communications: Theory and Applications." 2017, CRC Press. p. 337-372.

[9] Burroughes, J., et al., "Light-emitting diodes based on conjugated polymers." in nature, 1990. 347(6293): p. 539.

[10] Tang, C.W. and S.A. VanSlyke, "Organic electroluminescent diodes." in Applied Physics Letters, 1987. 51(12): p. 913-915.

[11] Thejokalyani, N. and S. Dhoble, "Novel approaches for energy efficient solid state lighting by RGB organic light emitting diodes-A review." in Renewable and Sustainable Energy Reviews, 2014. 32: p. 448-467.

[12] Lee, K.-Y., et al., "A new compensation method for emission degradation in an AMOLED display via an external algorithm, new pixel circuit, and models of prior measurements." J. of Display Tech., 2014. 10(3): p. 189197.

[13] Haigh, P.A., et al., "A 20-Mb/s VLC link with a polymer LED and a multilayer perceptron equalizer." in IEEE Photonics Techn. Letters, 2014. 26(19): p. 1975-1978.

[14] Le, S.T., et al., "10 Mb/s visible light transmission system using a polymer light-emitting diode with orthogonal frequency division multiplexing." in Optics Letters, 2014. 39(13): p. 3876-3879.

[15] Chen, H., et al., "A 51.6 Mbps Experimental VLC System Using A Monochromic Organic LED." in IEEE Photonics Journal, 2017.

[16] Baeg, K.J., et al., "Organic light detectors: photodiodes and phototransistors." Advanced Materials, 2013. 25(31): p. 4267-4295.

[17] Tedde, S.F., "Design, fabrication and characterization of organic photodiodes for industrial and medical applications." 2010, Technische Universität München.

[18] Boerner, H. "OLEDs for lighting. in Organic Optoelectronics and Photonics II." 2006. Intern. Society for Optics and Photonics.

[19] Haigh, P., "Using Equalizers to Increase Data Rates in Organic Photonic Devices for Visible Light Communications Systems." 2014, University of Northumbria.

[20] Shinar, R. and J. Shinar, "Organic electronics in sensors and biotechnology." 2009: McGraw-Hill, Inc.

[21] Fontanella, J., C. Andeen, and D. Schuele, "Pressure and Temperature Derivatives of the Low-Frequency Dielectric Constants of LiF, NaF, $\mathrm{NaCl}, \mathrm{NaBr}, \mathrm{KCl}$, and KBr." in Physical review B, 1972. 6(2): p. 582.

[22] Chvojka, P., et al. "Characterization of the organic LED based visible light communications." in Communication Systems, Networks and Digital Signal Processing (CSNDSP), 2016 10th Intern. Symposium on. 2016. IEEE.

[23] Haigh, P., et al. "Organic visible light communications: Methods to achieve $10 \mathrm{Mb} / \mathrm{s} . "$ in Photonics Conference (IPC), 2017 IEEE. 2017. IEEE. 
[24] Le Minh, H., et al. "Equalization for organic light emitting diodes in visible light communications." in GLOBECOM Workshops (GC Wkshps), 2011 IEEE. 2011. IEEE.

[25] Chen, H. and Z. Xu, "OLED Panel Radiation Pattern and Its Impact on VLC Channel Characteristics." IEEE Photonics Journal, 2017.

[26] James, C., "Fundamentals of Linear Electronics: Integrated and Discrete." Cengage Learning, 2001.

[27] Zvanovec, S., et al., "Visible light communications towards 5G." Radioengineering, 2015. 24(1): p. 1-9.

[28] Park, J.H., et al., "Roles of interlayers in efficient organic photovoltaic devices." Macromolecular rapid communications, 2010. 31(24): p. 2095 2108

[29] Chvojka, P., et al., "Analysis of nonline-of-sight visible light communications." Optical Engineering, 2017. 56(11): p. 116116.

[30] Rajbhandari, S., "Application of wavelets and artificial neural network for indoor optical wireless communication systems." 2010, Northumbria University.

[31] Kahn, J.M. and J.R. Barry, "Wireless infrared communications." Proceedings of the IEEE, 1997. 85(2): p. 265-298.

[32] Haigh, P.A., et al., "Exploiting equalization techniques for improving data rates in organic optoelectronic devices for visible light communications." Journal of Lightwave technology, 2012. 30(19): p. 3081-3088.

[33] Lee, K. and H. Park, "Modulations for visible light communications with dimming control." IEEE photonics technology letters, 2011. 23(16): p. 1136-1138.

[34] Wang, Z., et al., "Performance of dimming control scheme in visible light communication system." Optics express, 2012. 20(17): p. 18861-18868.

[35] Lee, K. and H. Park. "Channel model and modulation schemes for visible light communications." in Circuits and Systems (MWSCAS), 2011 IEEE 54th International Midwest Symposium on. 2011. IEEE.

[36] Zhao, J. and A.D. Ellis. "A novel optical fast OFDM with reduced channel spacing equal to half of the symbol rate per carrier." in Optical Fiber Communication (OFC), collocated National Fiber Optic Engineers Conference, 2010 Conference on (OFC/NFOEC). 2010. IEEE.

[37] Yamamoto, S., et al., "Achievement of subchannel frequency spacing less than symbol rate and improvement of dispersion tolerance in optical OFDM transmission." Journal of Lightwave Technology, 2010. 28(1): p. 157-163.

[38] Bosco, G., et al., "On the performance of Nyquist-WDM terabit superchannels based on PM-BPSK, PM-QPSK, PM-8QAM or PM16QAM subcarriers." Journal of Lightwave Technology, 2011. 29(1): p. 53-61

[39] Peng, W.-R., et al., "Spectrally efficient direct-detected OFDM transmission incorporating a tunable frequency gap and an iterative detection techniques." Journal of Lightwave Technology, 2009. 27(24): p. 5723-5735.

[40] Darwazeh, I., et al., "Optical SEFDM system; bandwidth saving using non-orthogonal sub-carriers." IEEE Photonics Technology Letters, 2014. 26(4): p. 352-355.

[41] Wu, F., et al., "Performance comparison of OFDM signal and CAP signal over high capacity RGB-LED-based WDM visible light communication." IEEE Photonics Journal, 2013. 5(4): p. 7901507-7901507.

[42] Haigh, P.A., et al., "Multi-band carrier-less amplitude and phase modulation for bandlimited visible light communications systems." IEEE Wireless Communications, 2015. 22(2): p. 46-53.
[43] Haigh, P.A., et al., "A multi-CAP visible-light communications system with $4.85-\mathrm{b} / \mathrm{s} / \mathrm{Hz}$ spectral efficiency." IEEE J. on Selected Areas in Communications, 2015. 33(9): p. 1771-1779.

[44] Chvojka, P., et al. "Variable m-CAP for bandlimited visible ligh communications." in Communications Workshops (ICC Workshops), 2017 IEEE Intern. Conference on. 2017. IEEE.

[45] Bandara, K., P. Niroopan, and Y.-H. Chung. "PAPR reduced OFDM visible light communication using exponential nonlinear companding." in Microwaves, Communications, Antennas and Electronics Systems (COMCAS), 2013 IEEE International Conference on. 2013. IEEE.

[46] Zhang, X., et al. "An advanced A-law companding algorithm in VLCOFDM." in Consumer Electronics (GCCE), 2014 IEEE 3rd Global Conference on. 2014. IEEE.

[47] Singh, A., A. Jain, and P. Vyavahare. "A study of peak to average powe ratio for different companding techniques in VLC-OFDM system." in Advances in Computing, Communications and Informatics (ICACCI), 2016 International Conference on. 2016. IEEE.

[48] Ghassemlooy, Z., C. Ma, and S. Guo, "PAPR reduction scheme for ACOOFDM based visible light communication systems." Optics Communications, 2017. 383: p. 75-80.

[49] Agrawal, G.P., "Fiber-optic communication systems." Vol. 222. 2012: John Wiley \& Sons

[50] Haigh, P.A., Z. Ghassemlooy, and I. Papakonstantinou, "1.4-Mb/s white organic LED transmission system using discrete multitone modulation." IEEE Photonics Technology Letters, 2013. 25(6): p. 615-618.

[51] Ghassemlooy, Z. "OLED-based visible light communications." in Photonics Society Summer Topical Meeting Series, 2012 IEEE. 2012. IEEE

[52] Haigh, P.A., et al., "Visible light communications: real time $10 \mathrm{Mb} / \mathrm{s}$ link with a low bandwidth polymer light-emitting diode." Optics express, 2014. 22(3): p. 2830-2838

[53] Haigh, P.A., et al., "Wavelength-multiplexed polymer LEDs: Towards 55 $\mathrm{Mb} / \mathrm{s}$ organic visible light communications." IEEE Journal on Selected Areas in Communications, 2015. 33(9): p. 1819-1828.

[54] Ghassemlooy, Z., et al., "Visible Light Communications: 3.75 Mb/s Data Rate with a $160 \mathrm{kHz}$ BW Organic Photodetector and ANN Equalization." Photonics Research, Vol. 1, Issue 2, pp. 65-68 (2013), 2013.

[55] Haigh, P.A., et al., "A 1-Mb/s visible light communications link with low bandwidth organic components." IEEE Photonics Technology Letters, 2014. 26(13): p. 1295-1298.

[56] Chen, H., et al. "A 1.9 Mbps OFDM-based all-organic visible ligh communication system." in Communication Systems (ICCS), 2016 IEEE Intern. Conference on. 2016. IEEE.

[57] Li, X., et al. "Volterra-based nonlinear equalization for nonlinearity mitigation in organic VLC." in Wireless Communications and Mobile Computing Conference (IWCMC), 2017 13th International. 2017. IEEE.

[58] Zhang, S., et al., "Organic solar cells as high-speed data detectors for visible light communication." Optica, 2015. 2(7): p. 607-610.

[59] Sarwar, R., et al. "Visible light communication using a solar-panel receiver." in Optical Communications and Networks (ICOCN), 2017 16th Intern. Conference on. 2017. IEEE.

[60] Malik, B. and X. Zhang. "Solar panel receiver system implementation for visible light communication." in Electronics, Circuits, and Systems (ICECS), 2015 IEEE International Conference on. 2015. IEEE. 